\title{
Automatic Tracking of Flying Vehicles Using Geodesic Snakes and Kalman Filtering
}

\author{
Amir Betser, Patricio Vela $\dagger$, Allen Tannenbaum \\ School of Electrical and Computer Engineering \\ Georgia Institute of Technology \\ Atlanta, GA, 30332-0250, USA \\ email: amir@ece.gatech.edu, pvela@ece.gatech.edu, tannenba@ece.gatech.edu
}

\begin{abstract}
This paper describes a tracking algorithm relying on active contours for target extraction and an extended Kalman filter for relative pose estimation. This work represents the first step towards treating the general problem for the control of several unmanned autonomous vehicles flying in formation using only local visual information. In particular, we only allow on-board passive sensing. The problem is an excellent paradigm for studying the use of visual information in a feedback loop, the central theme of controlled active vision.
\end{abstract}

\section{INTRODUCTION}

In this paper, we consider the use of geometric active contours for segmentation and Kalman filtering for the problem of tracking flying vehicles. Tracking is a basic control problem whereby a system's output is to follow or track a reference signal, or equivalently a system's tracking error should be as small as possible relative to some well-defined criterion (say energy, power, peak value, etc.). Although tracking in the presence of a disturbance is a classical control issue, the problem at hand is very difficult and challenging because of the highly uncertain nature of the disturbance.

We should note that the problem of visual tracking differs from standard tracking problems in that the feedback signal is measured using imaging sensors. In particular, it has to be extracted via computer vision algorithms and interpreted by a reasoning algorithm before being used in the control loop. Furthermore, the response speed is a critical aspect. Consequently, from the control point of view, we have a tracking problem in the presence of a highly uncertain disturbance which we want to attenuate. Note that the uncertainty is due to the sensor noise (classical), the algorithmic component described above (uncertainty in extracted features, likelihood of various hypotheses, etc.), and modelling uncertainty.

The problem we will consider here is the tracking of a leading Unmanned Aerial Vehicle (UAV) (the Leader) by another UAV (the Follower) without communication between the two vehicles. Sensing, for the Follower, is

$\dagger$ Corresponding author.

This work was supported by AFOSR and MURI. accomplished completely on-board and is passive. Such a problem is considered in Sattigeri et al [21]. However in this work the authors rely on the existence of relative range and line of sight measurements, which are not available in the problem we wish to solve. Consequently, vision-based estimation of the relative range and line of sight will be introduced. The line of sight angle is readily computable from the image itself. Range, on the other hand, is not generically observable from the image sequence alone and additional processing is required [1], [2].

The majority of vision-based techniques for range or depth estimation result in dense range-maps. Dense rangemaps may be estimated using optical flow [3], [24] or optical differentiation [6]. Harding and Lane [8] compute depth by solving the inverse image projection using multiple views. A Minimum Descriptor Length approach to optical flow computation and range estimation is given in [17]. PDE based methods for solving the range problem can be found in [7]. Another approach couples a nonlinear observer with the tracking of various geometric objects (lines, curves, etc.) across multiple frames [11]. Murphey et al [19] describe a "depth-finder" algorithm for planar motion. Many of these approaches are not real-time implementable or rely on static scenery.

There are some methods that deal with tracking a coherent object across multiple frames for range estimation. Avidan and Shashua [2] utilize trajectory triangulation to solve for the relative range. Stein et al [25] work out the geometry of vision for planar vehicles and compute the optimal sampling rate for estimation of range. These techniques are limited by model assumptions that need not hold in our case.

Much of the work regarding range estimation from bearings only information is relevant to the problem at hand. The bearings-only problem deals with range estimation using passively obtained bearing data (typically from sonar). Huster and Rock [10] examine the real-time implementation of the extended Kalman filter found in [1]. Other methods incorporate particle filters [13], multiple model hypotheses [15], or bias correction [18] to overcome the inherent limitations found in [1]. Much of the limitations can be traced 


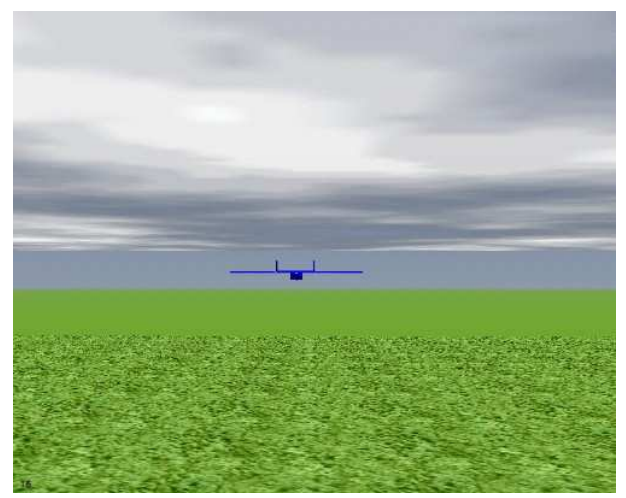

Fig. 1. Sample Simulation Image

to the paucity of sensor information. In our vision-based implementation, additional image information is available for improvement of the estimation process.

The purpose of this paper is to focus on proof-of-concept simulations implementing active contour tracking with an extended Kalman filter model for robust estimation of range and line of sight. Once proven, the next step is integration into a hardware-in-the-loop simulation [12] followed by actual deployment on an experimental setup. A sample frame $^{1}$ of the simulation is found in Figure 1. Such images will be inputs to the image processing algorithm.

\section{Object Tracking Via Active Contours}

This section reviews snakes or active contours and their evolution based on principles from curvature driven flows and the calculus of variations. Active contours may be regarded as autonomous processes which employ image coherence in order to track various features of interest over time. Such deformable contours have the ability to conform to various object shapes and motions, making them ideal for segmentation, edge detection, shape modelling, and visual tracking. In this paper, we will use active contours to dynamically segment a sequence of images, and then apply our estimation algorithms to the corresponding binary images.

The deformable contour model described in [14], [5] will be one of our key techniques for tracking. The method is based on Euclidean curve shortening evolution and on the theory of conformal metrics. The Euclidean curve shortening evolution defines the gradient direction whereby a given curve is shrinking most rapidly relative to Euclidean arclength. The Euclidean arc-length is modified by a conformal factor adapted to the desired features of interest. Under the corresponding gradient evolution equations, the features to capture lie at the bottom of a potential well to which the initial contour will flow. Level set methods allow fast robust implementations of the model.

In [4], [16], the authors introduce in a snake model based on the level set formulation of the Euclidean curve

\footnotetext{
${ }^{1}$ courtesy Prof. Eric Johnson.
}

shortening equation. More precisely, the model is

$$
\frac{\partial \Psi}{\partial t}=\phi(x, y)\|\nabla \Psi\|\left(\operatorname{div}\left(\frac{\nabla \Psi}{\|\nabla \Psi\|}\right)+\nu\right) .
$$

Here the function $\phi(x, y)$ depends on the given image and is used as a "stopping term." For example, the term $\phi(x, y)$ may be chosen to be small near an edge, and so acts to stop the evolution when the contour gets close to an edge. One may take [4], [16]

$$
\phi:=\frac{1}{1+\left\|\nabla G_{\sigma} * I\right\|^{2}},
$$

where $I$ is the (grey-scale) image and $G_{\sigma}$ is a Gaussian (smoothing filter) filter. The function $\Psi(x, y, t)$ evolves in (1) according to the associated level set flow for planar curve evolution in the normal direction with speed a function of curvature which was introduced in [20], [22].

It is important to note that the Euclidean curve shortening part of this evolution, namely

$$
\frac{\partial \Psi}{\partial t}=\|\nabla \Psi\| \operatorname{div}\left(\frac{\nabla \Psi}{\|\nabla \Psi\|}\right)
$$

is derived as a gradient flow for shrinking the perimeter as quickly as possible. As is explained in [4], the constant inflation term $\nu$ is added in (1) in order to keep the evolution moving in the proper direction. Note that we are taking $\Psi$ to be negative in the interior and positive in the exterior of the zero level set.

To modify the model (1) in a manner consistent with curve shortening flow, consider the conformal length metric

$$
d s_{\phi}=\left(x_{p}^{2}+y_{p}^{2}\right)^{\frac{1}{2}} \phi d p,
$$

where $\phi(x, y)$ is a positive differentiable function and the curve in question is parametrized by $p$, e.g., $C=$ $(x(p), y(p))^{T}$. The standard Euclidean metric is recovered with $\phi=1$. To compute the corresponding gradient flow for shortening length relative to the metric $d s_{\phi}$, define the modified length functional $L_{\phi}(t)$,

$$
L_{\phi}(t):=\int_{0}^{1} d s_{\phi}=\int_{0}^{1}\left\|\frac{\partial C}{\partial p}\right\| \phi d p .
$$

Taking the first variation of the modified length function and using integration by parts [14],

$$
L_{\phi}^{\prime}(t)=-\int_{0}^{L_{\phi}(t)}\left\langle\frac{\partial C}{\partial t}, \phi \kappa \overrightarrow{\mathcal{N}}-(\nabla \phi \cdot \overrightarrow{\mathcal{N}}) \overrightarrow{\mathcal{N}}\right\rangle
$$

The direction in which the $L_{\phi}$ perimeter is shrinking as fast as possible is given by

$$
\frac{\partial C}{\partial t}=(\phi \kappa-(\nabla \phi \cdot \overrightarrow{\mathcal{N}})) \overrightarrow{\mathcal{N}}
$$

precisely the gradient flow corresponding to the minimization of the length functional $L_{\phi}$.

The level set version of this is

$$
\frac{\partial \Psi}{\partial t}=\phi\|\nabla \Psi\| \operatorname{div}\left(\frac{\nabla \Psi}{\|\nabla \Psi\|}\right)+\nabla \phi \cdot \nabla \Psi .
$$


The evolution of Equation (5) should attract the contour very quickly to the feature lying at the bottom of the potential well determined by the conformal factor $\phi$. As in [4], [16], a constant inflation term $\nu$ may be added to obtain a modified model similar to (1),

$$
\frac{\partial \Psi}{\partial t}=\phi\|\nabla \Psi\|\left(\operatorname{div}\left(\frac{\nabla \Psi}{\|\nabla \Psi\|}\right)+\nu\right)+\nabla \phi \cdot \nabla \Psi .
$$

Notice that for $\phi$ as in (2), $\nabla \phi$ will look like a doublet near an edge. Of course, one may choose other candidates for $\phi$ in order to pick out alternative features.

We now have very fast implementations of these snake algorithms based on level set methods [20], [22]. The ability of the snakes to change topology and quickly capture desired features makes them an indispensable tool for our visual tracking algorithms. See also [26] for more details about this. We use such active contours to derive segmented data which then drives the estimation process.

\section{PROBLEM Formulation}

The problem consists of two planar UAV's, one of which is labelled the Leader and the other Follower. The Leader is following an unknown trajectory relative to which the Follower must track, c.f., Figure 2. Available to the Follower are measurements of its own state (configuration, velocity, and acceleration) and the image obtained from a fixed, forward-pointing, on-board, monocular camera system.

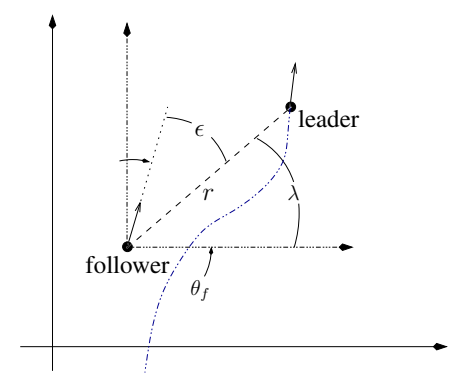

Fig. 2. Leader-Follower Confi guration

The complete closed loop system is summarized by the block diagram of Figure 3. The Image Processing and Computer Vision block produces "measurements" (which will described in the sequel) to the Estimation block, which is based on an Extended Kalman Filter. The Estimation block calculates the relative range, the line of sight (LOS), and the LOS rate between the Leader and the Follower. These parameters are used by the Guidance block to produce the velocity and acceleration commands for the vehicle's inner controller loop.

The measurement input to the Kalman filter are two angles. The first angle is the lead angle $\epsilon$, and the second is the maximum angle subtended by the Leader in the image plane $\alpha$. The maximum angle subtended can be determined quite easily from the active contour tracking the Leader on the image plane.

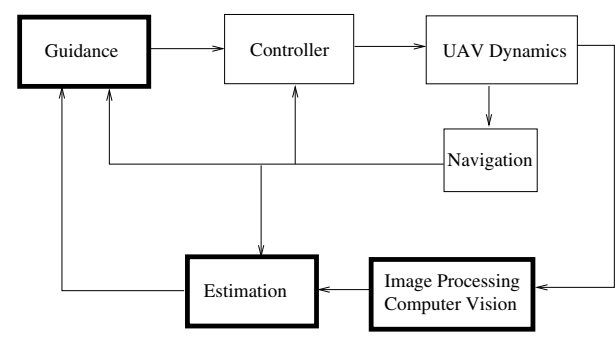

Fig. 3. System Block Diagram

\section{The Estimation Process}

In this section we will describe a modified algorithm for estimating the relative range $r$, LOS angle $\lambda$, and the LOS rate $\dot{\lambda}$ using the visual information obtained from a fixed, forward-pointing on-board camera. The algorithm is an extension of the extended Kalman Filter found in Aidala and Hammel [1]. The extended Kalman filter model equations involving relative range and LOS are

$$
\dot{x}(t)=\left[\begin{array}{c}
-2 x_{1}(t) x_{2}(t)+x_{4}(t) a_{N}^{L O S}(t) \\
x_{1}^{2}(t)-x_{2}^{2}(t)+x_{4}(t) a_{T}^{L O S}(t) \\
x_{1}(t) \\
-x_{2}(t) x_{4}(t)
\end{array}\right],
$$

with the filter states

$$
x(t)=\left[\begin{array}{c}
x_{1}(t) \\
x_{2}(t) \\
x_{3}(t) \\
x_{4}(t)
\end{array}\right]=\left[\begin{array}{c}
\dot{\lambda} \\
\dot{r} / r \\
\lambda \\
1 / r
\end{array}\right],
$$

where $a_{N}^{L O S}$ and $a_{T}^{L O S}$ are the relative acceleration normal and tangent to the LOS, respectively. The available measurement is the LOS angle as obtained from the lead angle,

$$
\lambda_{m}(t)=\theta_{f}(t)-\epsilon(t)+\omega_{\lambda}
$$

where $\theta_{f}$ is the heading of the Follower, and $\omega_{\lambda}$ is measurement noise.

Aidala and Hammel [1] demonstrated that the range state is unobservable except during certain maneuvers. Furthermore, should the Leader accelerate or maneuver in any way, the extended Kalman filter would diverge. Work has gone into understanding the optimal maneuvers for range estimation [9], and into extensions to the Kalman filter framework for overcoming the effects of Leader acceleration [15], [18].

a) Extended model.: The Kalman filter equations (7)(9) do not completely utilize the information provided by the imaging camera. In particular, the captured image of the Leader provides indirect observation of the range,

$$
r=\frac{\delta}{2 \tan \left(\frac{1}{2} \alpha\right)} .
$$

The parameter $\delta$ is the nominal length of the Leader, an unknown quantity. This length is defined to be the longest axis of the plane (typically along the wing). By measuring the angle subtended by the Leader in the image plane, the unobservable state $r$ may be rendered observable during 
Follower motion. Consequently, adding the fifth state $x_{5} \equiv$ $\alpha$, with the dynamics,

$$
\dot{x}_{5}=-x_{2} \sin \left(x_{5}\right) .
$$

should improve the range estimates and provide a level of robustness to the estimation framework. This new state now provides range information during maneuvers tangent to the line of sight, whereas the original Kalman filter did not. Consequently, any acceleration by the Follower will provide range information to the Kalman filter.

Unfortunately, motion by the Leader still causes problems for the estimation process. Although the open loop estimation process may suffer as a result, range limits introduced in the closed loop system curtail the potential consequences. The range limits are obtained from Equation (10) by assuming that the Leader size $\delta$ lies within a particular range $\left[\delta_{\text {min }}, \delta_{\max }\right]$, which does not severly limit its possible size.

\section{A. Open Loop Results}

The equations (7)-(11) were implemented in a discrete extended Kalman filter with the addition of process noise to the model equations [27]. Using the original extended Kalman filter equations (7)-(9) the range is observable only during certain Follower maneuvers [1] so long as the Leader does not accelerate. Figure 4 depicts such a scenario. Both Kalman filters are capable of tracking the correct range, however, it can be seen that the vision-based estimator has improved convergence properties relative to the bearingsonly estimator.

Furthermore, Figure 5 depicts the estimate degradation due to Leader acceleration (begins at $t=80 \mathrm{~s}$ ). The bearings-only estimator can no longer track the relative range and diverges, whereas the vision-based estimator resettles into a small steady-state error once the acceleration stops $(t=90 \mathrm{~s})$. This demonstrates the improved robustness due to the additional vision-based measurement.

\section{GUIDANCE AND CONTROL}

This section provides an algorithm for tracking the Leader UAV so as to move from estimation to feedback control. The algorithm is based on Proportional Navigation and LOS guidance laws [23]. Referring to the block diagram of Figure 3, the Guidance block receives inputs from the Estimation block and two commands: the desired relative range $r^{*}$ and the desired relative (lead) angle $\epsilon^{*}$. The output of the algorithm are the acceleration commands to the inner control block of the UAV. Control acceleration for the autonomous vehicle is decomposed into normal and tangential acceleration components, $a_{N}$ and $a_{T}$.

b) Computation of $a_{N}$ and $a_{T} .:$ The purpose of the input $a_{N}$ is to maintain the desired LOS angle beween the Leader and Follower. Using the estimated values for the LOS and the LOS rate, define

$$
a_{N}=n v_{f}\left(K_{N} \hat{x}_{1}+\left(1-K_{N}\right) e_{\lambda}\right)
$$

Where $n$ is the proportional navigation constant, $K_{N}$ is a parameter in the range $[0,1], v_{f}$ is the forward velocity of the Follower, and $e_{\lambda} \equiv \epsilon^{*}-\left(\theta_{f}-\hat{x}_{3}\right)$.

The role of $a_{T}$ is to track the desired relative range between the Leader and Follower. Consequently $a_{T}$ is a function of the range error, $e_{r}=r^{*}-1 / \hat{x}_{4}$, described by the control loop depicted in Figure 6, where $v_{n o m}$ is the nominal forward velocity of the Follower.

\section{A. Closed Loop Results}

By incorporating the guidance and control algorithms into the estimation process described in Section IV, it is possible to achieve the desired trajectory tracking goal of the Follower. Simulation results of such a scenario are depicted in Figure 7. The Follower maintains a steady velocity with occasional changes in bearing and/or velocity. The visual processing and estimation algorithms work together to estimate the proper feedback for the guidance and control laws. Steady-state error is within $10 \%$.

\section{Conclusions and Future Research}

The extended Kalman filter found in [1] was improved by introducing additional image information available to vehicles with a fixed forward-pointing monocular camera. Active contours were used to track the follower in the image plane and provide the Kalman filter with the required input. Successful simulation of the Kalman filter has provided

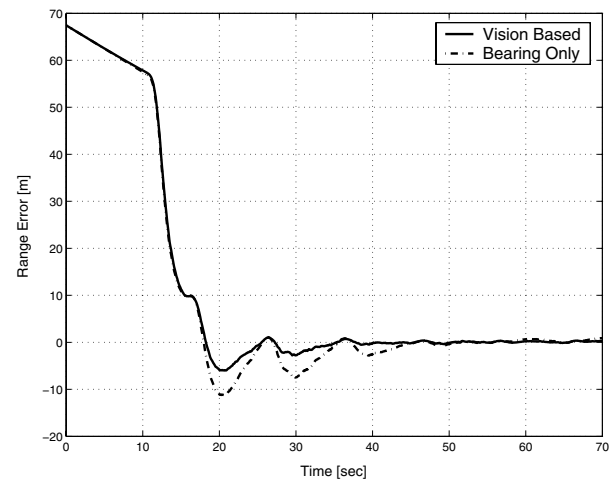

Fig. 4. Open Loop Graph

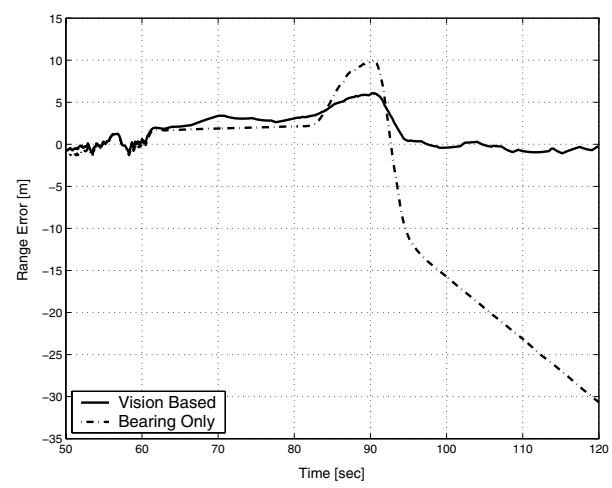

Fig. 5. Open Loop Graph 


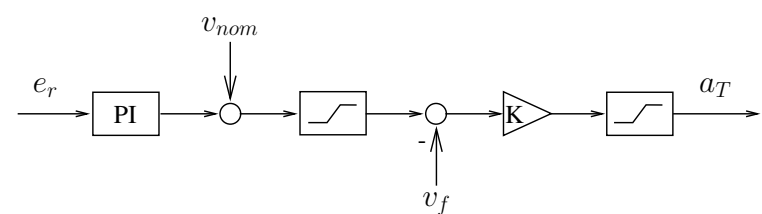

Fig. 6. Axial acceleration command control loop.

the proof-of-concept needed to continue investigation into real-time implementation of range estimation for automatic tracking of flying vehicles. To do so, the filter state will have to incorporate the additional angle measurement found in the equivalent 3D range estimation problem (using spherical coordinates). Furthermore, additional research should go into reducing the sensitivity of the extended Kalman filter to target acceleration.

The next step is to integrate the vision-based range estimation strategy with the formation flight control algorithm of [21] within the simulation environment discussed in [12] for hardware-in-the-loop simulation and validation. Successful integration will provide us with the platform to examine more complex vision-based UAV navigation scenarios.

c) Acknowledgements.: The authors are indebted to Dr. George Hexner for his advice regarding the bearingsonly Kalman filter equations.

\section{REFERENCES}

[1] V. J. Aidala and S. E. Hammel. Utilization of modifi ed polar coordinates for bearings-only tracking. IEEE Trans. Aut. Cont., 28:283-294, 1983.

[2] S. Avidan and A. Shashua. Trajectory triangulation: 3D reconstruction of moving points from a monocular image sequence. IEEE Trans. Patt. Anal. Mach. Int., 22(4):348-357, 2000.

[3] J. Barron, W.K.J Ngai, and H. Spies. Quantitative Depth Recovery from Time-Varying Optical Flow in a Kalman Filter Framework, pages 346-355. Lect. Notes in Comp. Sci. 2003.

[4] V. Caselles, F. Catte, T. Coll, , and F. Dibos. A geometric model for active contours in image processing. Numerische Mathematik, 66:1-31, 1993

[5] V. Caselles, R. Kimmel, and G. Saprio. Geodesic active contours. Int. J. Comp. Vis., 13:5-22, 1997.

[6] H. Farid and E.P. Simoncelli. Range estimation by optical differentiation. J. Opt. Soc. Am. A, 15(7):1777-1786, 1998.

[7] O. Faugeras and R. Keriven. Variational principles, surface evolution, PDEs, level set methods, and the stereo problem. IEEE Trans. Im. Proc., 7(3):336-344, 1998.

[8] C.M. Harding and R.G. Lane. Passive navigation from image sequences by use of a volumetric approach. J. Opt. Soc. Am. A, 19(2):295-305, 2002.

[9] J.P. Helferty and D.R. Mudgett. Optimal observer trajectories for bearings only tracking by minimizing the trace of the Cramer-Rao lower bound. In Proc. IEEE Conf. Dec. and Cont., pages 936-939, 1993.

[10] A. Huster and S.M. Rock. Relative position estimation for manipulation tasks by fusing vision and inertial measurements. In Proc. MTS/IEEE Oceans Conf., volume 2, pages 1025-1031, Honolulu, HI, 2001

[11] M. Jankovic and B.K. Ghosh. Visually guided ranging from observation of points, lines, and curves via an identifi er based nonlinear observer. Systems \& Control Letters, 25:63-73, 1995.

[12] E. Johnson and S. Mishra. Simulation for the development of an experimental UAV. In Proc. AIAA Mod. and Sim. Tech. Conf., 2002.

[13] R. Karlsson and F. Gussafsson. Range estimtaion using angle-only target tracking with particle fi lters. In Proc. Am. Cont. Conf., 2001.

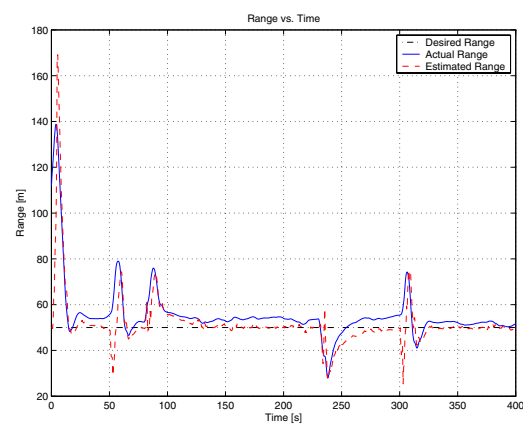

(a) Range vs. Time.

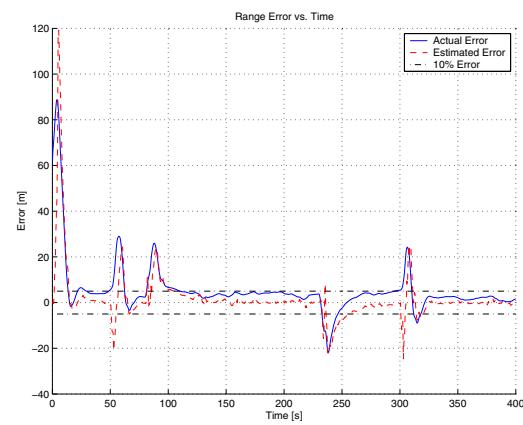

(b) Range Error vs. Time.

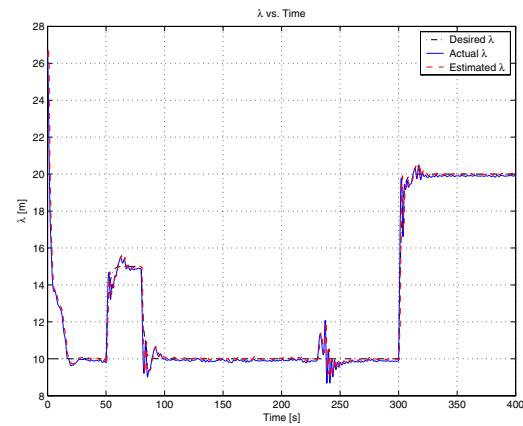

(c) $\lambda$ vs. Time

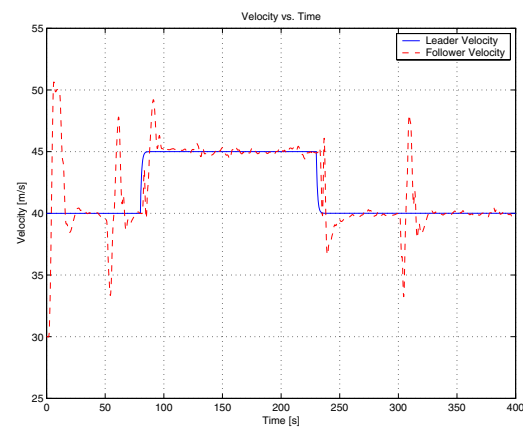

(d) Follower velocity vs. Time.

Fig. 7. Closed-loop Leader-Follower scenario. 
[14] S. Kichenassamy, A. Kumar, P. Olver, A. Tannenbaum, and A. Yezzi. Conformal curvature fbws: From phase transitions to active vision. Arch. Rat. Mech. Anal., 134:275-301, 1996.

[15] T. R. Kronhamn. Angle-only tracking of maneuvering targets using adaptive-IMM multiple range models. In $R A D A R$, pages $310-314$, 2002.

[16] R. Malladi, J. Sethian, and B. Vermuri. Shape modelling with front propagation: A level set approach. IEEE PAMI, 17:158-175, 1995.

[17] A. Mitiche and S. Hadjres. MDL estimation of a dense map of relative depth and 3D motion from a temporal sequence of images. Patt. Anal. Appl., 6:78-87, 2003.

[18] M.J. Moorman and T.E. Bullock. A new estimator for passive tracking of maneuvering targets. In Proc. IEEE Conf. Cont. App., volume 2, pages 1122-1127, 1992.

[19] Y.L. Murphey, J. Chen, J. Crossman, J. Zhang, P. Richardson, and L. Sieh. DepthFinder: A real-time depth detection system for aided driving. In Int. Veh. Symp., 2000.

[20] S. J. Osher and J. A. Sethian. Fronts propagation with curvature dependent speed: Algorithms based on Hamilton-Jacobi formulations. J. Comp. Phys., 79:12-49, 1988.

[21] R. Sattigeri, A. J. Calise, and J. H. Evers. An adaptive approach to vision-based formation control. In AIAA GNC Conf. and Exh., 2003.

[22] J. A. Sethian. Curvature and the evolution of fronts. Commun. Math. Phys., 101:487-499, 1985.

[23] N. Shneydor. Missile Guidance and Pursuit - Kinematics, Dynamics, and Control. Horwood Pub., Chichester, 1998.

[24] B. Sinopoly, M. Micheli, G. Donato, and T. J. Koo. Vision-based navigation for an unmanned aerial vehicle. In Proc. of the IEEE Int. Conf. on Int. Rob. and Aut., pages 1757-1764, Korea, 2001.

[25] G.P. Stein, O. Mano, and A. Shashua. Vision-based ACC with a single camera: Bounds on range and range rate accuracy. In Int. Veh. Symp., 2003.

[26] A. Tannenbaum and A. Yezzi. Visual tracking, active vision, and gradient flows, volume 237 of Lec. Notes in Cont. and Inf. Sci. Springer-Verlag, 1998.

[27] P. Zarchan and H. Mussof. Fundamentals of Kalman fi ltering - a practical approach. In P. Zarchan, editor, Progress in Astronautics and Aeronautics. Am. Inst. of Aer. and Astro., Inc., 1998. 